\title{
BMJ Open Understanding the underlying drivers of obesity in Africa: a scoping review protocol
}

\author{
Anelisa Jaca (1) , ${ }^{1}$ Chinwe Iwu (1) , ${ }^{2}$ Solange Durão, ${ }^{1}$ Adelheid W Onyango, ${ }^{3}$ \\ Charles Shey Wiysonge (1) ${ }^{1,4,5}$
}

To cite: Jaca A, Iwu C, Durão S, et al. Understanding the underlying drivers of obesity in Africa: a scoping review protocol. BMJ Open 2020;10:e040940. doi:10.1136/ bmjopen-2020-040940

- Prepublication history for this paper is available online. To view these files, please visit the journal online (http://dx.doi org/10.1136/bmjopen-2020040940).

Received 27 May 2020 Revised 11 August 2020 Accepted 30 September 2020

Check for updates

(C) Author(s) (or their employer(s)) 2020. Re-use permitted under CC BY. Published by BMJ.

${ }^{1}$ Cochrane South Africa, South African Medical Research Council, Tygerberg, South Africa ${ }^{2}$ Department of Nursing and Midwifery, Faculty of Medicine and Health Sciences, Stellenbosch University, Cape Town, South Africa

${ }^{3}$ Department of Nutrition for Health and Development, World Health Organization, Brazzaville, Congo

${ }^{4}$ School of Public Health and Family Medicine, University of Cape Town, Cape Town, South Africa

${ }^{5}$ Department of Global Health, Stellenbosch University, Cape Town, South Africa

Correspondence to

Dr Anelisa Jaca;

anelisaj@gmail.com

\section{ABSTRACT}

Introduction The worldwide prevalence of obesity and overweight has doubled since 1980, such that approximately a third of the world's population is reported as obese or overweight. Obesity rates have increased in all ages and both sexes irrespective of geographical area, ethnicity or socioeconomic status. Due to the high prevalence, related health consequences and costs of childhood and adult obesity, there is a need to comprehensively identify and assess the major underlying drivers of obesity and overweight in the African context. Methods and analysis This scoping review will be carried out as per the methodological outline by Arksey and O'Malley. The search strategy will be developed and search performed in the Scopus and PubMed electronic databases. In the first search, we will identify concepts that are used as an equivalent to obesity and overweight. Subsequently, we will search for studies comprising of search terms on the underlying factors that drive the development of obesity and overweight. Lastly, we will check reference lists for additional publications. Abstracts and full-text studies will independently be screened by two authors.

Ethics and dissemination The proposed study will generate evidence from published data and hence does not require ethics approval. Evidence generated from this review will be disseminated through journal publications and conference presentations.

\section{INTRODUCTION}

Obesity is an intricate, multifactorial and preventable health issue currently affecting, together with overweight, over a third of the world's population. ${ }^{1}$ The prevalence of obesity is seen across all ages, populations, ethnic groups and socioeconomic status. ${ }^{2}$ Obesity was once considered a high-income country health problem but it is now also commonly seen in low-income and middle-income countries. ${ }^{34}$ In low-income countries, this condition is more predominant among middle-aged adults, especially women of childbearing age (15 and 49 years), while it affects individuals of all ages and genders in high-income countries. ${ }^{5}$ In 2016 , over 1.9 billion adults were overweight and of those individuals, more than 650 million were obese, worldwide. ${ }^{6}$ Approximately 40 million

\section{Strengths and limitations of this study}

This scoping review will synthesise evidence on all factors related to the drivers of obesity in Africa.

- This study will employ a rigorous methodology which involves an established research framework, a search strategy and a selection process.

- Our review will potentially not include all research papers related to this theme area as some may not be accessible or published.

children under the age of 5 years and 340 million adolescents were reported to be obese between 2016 and 2018, globally. ${ }^{7}$ The number of children, under the age of 5 years, suffering from obesity or overweight in Africa was estimated at 9.5 million in the year $2018 .^{8}$ It is estimated that $20 \%$ of the world's adult population will be obese and $38 \%$ overweight by 2030 if this health issue is not dealt with. ${ }^{1}$

The WHO defines obesity as a surplus of fat that can affect one's health. The commonly used measure of obesity is the body mass index (BMI), calculated by dividing the body weight in kilograms by the square of height in metres. ${ }^{9}$ The WHO defines a normal BMI range as 18.5 to 24.9 suggesting that individuals with BMI greater than $25 \mathrm{~kg} / \mathrm{m}^{2}$ and 30 $\mathrm{kg} / \mathrm{m}^{2}$ are considered overweight and obese, respectively. ${ }^{10}$ Obesity affects nearly all physiological functions of the body and hence increases the risk of developing conditions such as cardiovascular (eg, heart failure) and metabolic (type 2 diabetes mellitus, hypertension and stroke) diseases. ${ }^{10-12}$

Obesity is predominantly associated with the overconsumption of foods with high sugar, calories and fats through the overconsumption of processed, inexpensive and poor quality foods that are not nutrient dense. ${ }^{13}$ The excess food that is consumed is changed into triglycerides which are stored in adipose tissues, hence increasing body fat and ultimately resulting in weight gain. ${ }^{14}$ Additionally, the nutrition 
transition, which encompasses changes from predominantly plant-based diets to those predominantly meat-based, is highly associated with weight gain and illnesses related to it. ${ }^{16}{ }^{17}$ Therefore, healthy eating behaviours can play a major role in reducing the prevalence of obesity and its related diseases. ${ }^{18}$

Alongside the rise in consumption of processed foods there has also been an increase in sedentary behaviours in Africa. ${ }^{19}{ }^{20}$ This may have contributed to increased rates of obesity as weight gain is associated with a positive energy balance, that is, calorie intake is greater than expenditure. ${ }^{21}$ Literature has shown that obesity rates in adults are generally significantly higher in sedentary compared with active individuals. ${ }^{22} \mathrm{~A}$ decrease in daily energy expenditure is associated with weight gain while high levels of physical activity significantly mitigate weight gain. ${ }^{23} 24$ Physical activity is defined as any bodily movement that demands energy use, e.g., walking, cycling, sport activities and doing house chores. On the other hand, physical inactivity or sedentary behaviour is a lifestyle identified by an energy expenditure less than 1.5 metabolic equivalents while in a sitting, reclining or lying position. ${ }^{25}$ In addition to dietary and sedentary patterns, other contributing factors to the high prevalence of obesity include smoking, alcohol consumption, urbanisation and commonly used medications, namely, psychotropic medications, diabetic treatments, antihypertensives, steroid hormones and contraceptives. $^{26}$

\section{Study rationale}

There is growing acknowledgement that it will be challenging to practice healthy lifestyles unless we understand the factors that drive and contribute to unhealthy behaviours. Factors including diet, level of physical activity and age have been widely reported to influence obesity. Obesity increases with age which may be explained by decreases in physical activity and metabolic activity in older adults. The more we understand how these, or other factors drive obesity in Africa, the better we will be able to address it. Without proper understanding of the drivers of obesity in this setting, many individuals will not only suffer from obesity-related conditions but from costs sustained during treatment. Since obesity not only contributes to poor health but also affects people's psychological and social well-being, addressing it requires understanding and appreciating our biology, behaviour, environment and culture. There is a need to better understand the various factors that motivate our eating habits and sedentarity, driving obesity. To date, no synthesised evidence exists that investigated the underlying drivers of obesity in Africa. We will therefore conduct a narrative synthesis of the underlying drivers of obesity in the African setting.

\section{Study objectives}

The objective of this scoping review is to chart the existing evidence on the underlying drivers of obesity and overweight in the African context.

\section{Methodology}

A scoping review is used to gather emerging evidence in various fields with relevance to time, population (eg, country or context), source (eg, peer-reviewed or grey literature) and origin (eg, healthcare discipline or academic field). This study design is suitable for addressing broad concepts, questions that seek to assess the effects of interventions to inform practice, policy and research. We will thus conduct a scoping review, for studies published by end of March 2020, in order to map the crucial concepts and bring together the existing evidence on the underlying drivers of obesity in the African context. We will carry out this scoping review employing a predefined protocol as per the Arksey and O'Malley methodological outline. ${ }^{27}$ This outline includes identifying the research question, searching for relevant studies, selecting studies, charting and collating data, summarising and reporting results. This scoping review will be reported as per the Preferred Reporting Items for Systematic Reviews and Meta-Analyses extension for Scoping Reviews (PRISMA-ScR) checklist. ${ }^{28}$

\section{Defining the research question}

This scoping review will identify the drivers of obesity in the African setting. The research question: 'What are the underlying drivers of obesity among children and adults in Africa' was framed using the Population, Exposure, Concept and Context element (table 1).

\section{Inclusion criteria}

We will include studies of any design that focus on drivers or risk factors of obesity. These drivers may include dietary factors (eg, overconsumption of high-energy/low nutrient, processed and obesogenic foods), sedentary behaviours (eg, physical inactivity or increased sedentary behaviours), smoking status, alcohol consumption, urbanisation and commonly used medications (eg, psychotropic medications, diabetic treatments, antihypertensives, steroid hormones

Table 1 PECC element for defining the eligibility criteria of the studies for the research question

\begin{tabular}{|c|c|c|c|}
\hline Population & Exposure & Concept & Context \\
\hline
\end{tabular}

BMI, body mass index; PECC, Population, Exposure, Concept and Context. 
Table 2 Initial search strategy developed for PubMed for the scoping review on the underlying drivers of obesity and overweight among children and adults in Africa

\begin{tabular}{|c|c|}
\hline Search \# & Search texts and syntaxes \\
\hline \#1 & obesity OR obese OR overweight OR body mass index OR fat OR fatness \\
\hline \#2 & physical inactivity OR physically inactive OR sedentar* \\
\hline \#3 & 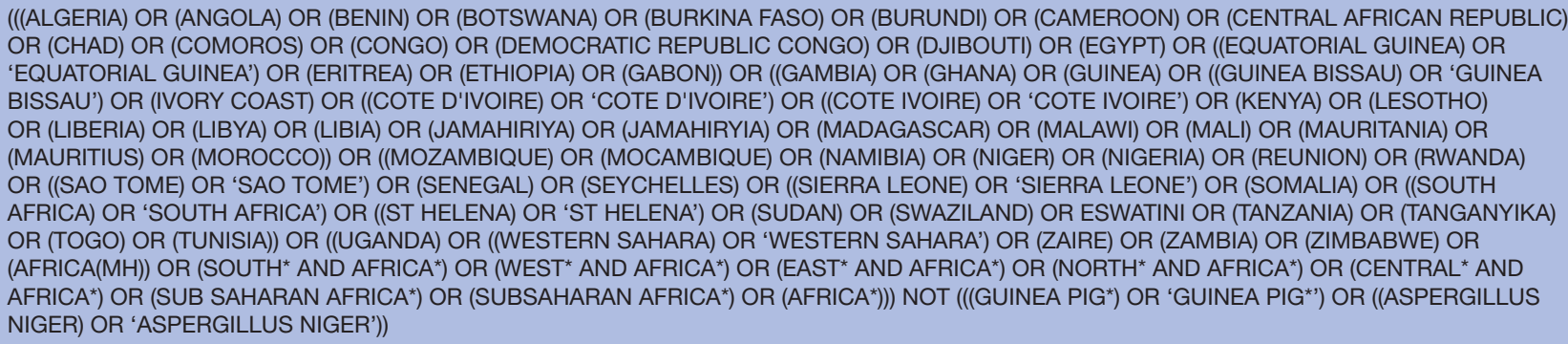 \\
\hline \#4 & \#1 AND \#2 AND \#3 \\
\hline
\end{tabular}

and contraceptives). Furthermore, the studies must have assessed and reported outcomes such as the type of food consumed, type of medication taken, sedentary behavioural patterns and prevalence of obesity and overweight. This review will include obese and overweight individuals of all ages, that is, children (between ages 2 and 12 years), adolescents (13 to 17 years), young adults (18 to 35 years), older adults (36 to 55 years) and the elderly ( 56 years and older) and both genders (male and female). We will define overweight adults as those with a BMI greater than or equal to $25 \mathrm{~kg} / \mathrm{m}^{2}$, while those with a BMI greater than or equal to $30 \mathrm{~kg} / \mathrm{m}^{2}$ will be considered obese. In children between the ages of 5 and 19 years, overweight will be defined as a BMI $>+1$ SD equivalent to $25 \mathrm{~kg} / \mathrm{m}^{2}$ while those with a $\mathrm{BMI}>+2$ SD equivalent to $30 \mathrm{~kg} / \mathrm{m}^{2}$ will be deemed obese. ${ }^{29}$

\section{Selection of studies relevant to the research question \\ Search strategy}

We will conduct a comprehensive literature search in Scopus and PubMed databases. No language or date restrictions will be used. In our search, we will combine the key terms such as 'obesity OR obese OR overweightness OR overweight OR weight gain' AND physical inactivity OR physically inactive OR sedentar* (table 2). The first 100 search results from each database will be reviewed by the researchers to assess validity of the search strategy. When agreement is reached about the initial search strategy, the first 200 abstracts will be screened by two authors (AJ and CI) on concepts potentially eligible for inclusion in the second search step. In the event of full agreement between the two authors on potentially eligible concepts, AJ will screen the rest of the abstracts. In the event of disagreement, CI will screen the abstracts until full agreement is reached. After all abstracts have been screened, AJ and CI will discuss all potentially eligible search terms and select words to be included in the subsequent search step. After having selected the different search terms, AJ and CI will develop a search query in PubMed and then apply it to other databases. Following the searches, the two authors will also search reference lists of relevant studies for other eligible articles.

\section{Screening and selection}

Two review authors (AJ and CI) will independently screen the titles and abstracts of articles identified in the search output for potentially eligible studies. The authors will obtain the full-text articles for any study that is considered potentially eligible and then independently appraise the full text of each potentially eligible study and classify it as either included or excluded. We will provide reasons for excluding potentially eligible studies from the review. Disagreements among the two authors during the screening and study selection process will be resolved through discussion and arbitration by a third author.

\section{Data charting and extraction}

Data will be extracted independently by two authors (AJ and CI) from the eligible studies and differences resolved by a third author (CSW). Data extracted will comprise study characteristics (year the study was conducted/published, country of origin, study design, number of study arms and comparators); participants (children and adults); setting (African countries) and drivers or risk factors of obesity. These will include demographic (age and gender), environmental (suburb and rural areas), food availability (high calories and energy dense food), socioeconomic (education level, employment status and income level), behavioural (eg, physical inactivity, diet and alcohol consumption) and medication-related drivers.

\section{Patient and public involvement}

This study will employ publicly available data and hence will not involve any patients and the public.

\section{Collating, summarizsing and reporting results of the review}

A PRISMA flow diagram will be used to report the final number of included studies in the review. We will synthesise study findings using narrative descriptions based on subjects that emerge from the extracted data. The results will be compared and consolidated through consensus between two authors (AJ and CI), where both quantitative and qualitative aspects of research evidence will be 
addressed. We will discuss and present data obtained from obesity-related outcomes reported in each study.

\section{Expert consultation}

In order to confirm our findings and interpretations, an expert in the field of nutrition and obesity will be approached for consultation.

\section{Ethics and dissemination}

The methodology applied to carry out this review will comprise reviewing and collecting evidence from openly available data; therefore, this study does not require ethical approval. This scoping review will be the first to collect evidence that has investigated the underlying drivers of obesity in Africa. By identifying gaps in the current body of literature, this study can influence forthcoming research on obesity in Africa. The results that will be generated from this review will be shared with the public through peer-reviewed publications and national and international conferences.

Twitter Anelisa Jaca @AnelisaJaca, Chinwe Iwu @chinwejaja and Charles Shey Wiysonge @CharlesShey

Acknowledgements We would like to acknowledge the South African Medical Research Council for proving the office facilities to undertake this project.

Contributors AJ wrote the first draft of the protocol. CI, SD, AW0 and CSW critically revised the intellectual content of the manuscript.

Funding World Health Organization Region for Africa (grant number: N/A). Competing interests None declared.

Patient and public involvement Patients and/or the public were not involved in the design, or conduct, or reporting or dissemination plans of this research.

Patient consent for publication Not required.

Provenance and peer review Not commissioned; externally peer reviewed.

Data availability statement The data that will be generated from this paper will be made publicly available.

Open access This is an open access article distributed in accordance with the Creative Commons Attribution 4.0 Unported (CC BY 4.0) license, which permits others to copy, redistribute, remix, transform and build upon this work for any purpose, provided the original work is properly cited, a link to the licence is given, and indication of whether changes were made. See: https://creativecommons.org/ licenses/by/4.0/.

\section{ORCID iDs}

Anelisa Jaca http://orcid.org/0000-0002-9814-8374

Chinwe Iwu http://orcid.org/0000-0003-0765-7497

Charles Shey Wiysonge http://orcid.org/0000-0002-1273-4779

\section{REFERENCES}

1 Hruby A, Hu FB, PhD M. The epidemiology of obesity: a big picture. Pharmacoeconomics 2015;33:673-89.

2 Ells LJ, Rees K, Brown T, et al. Interventions for treating children and adolescents with overweight and obesity: an overview of Cochrane reviews. Int J Obes 2018;42:1823-33.

3 Bleich SN, Vercammen KA, Zatz LY, et al. Review Interventions to prevent global childhood overweight and obesity : a systematic review. LANCET Diabetes Endocrinol 2017;8587:1-15.
4 Laws R, Campbell KJ, van der Pligt P, et al. The impact of interventions to prevent obesity or improve obesity related behaviours in children ( $0-5$ years) from socioeconomically disadvantaged and/or Indigenous families: a systematic review. BMC Public Health 2014;14:779.

5 Hutchesson MJ. De Jonge Mulock Houwer M, brown HM, LIM S, Moran LJ, Vincze L, et al. supporting women of childbearing age in the prevention and treatment of overweight and obesity: a scoping review of randomized control trials of behavioral interventions. BMC Womens Health 2020;20:1-15.

6 World Health Organization. Obesity and overweight [Internet], 2020. Available: https://www.who.int/news-room/fact-sheets/detail/ obesity-and-overweight [Accessed 2020 Apr 3].

7 Wordld Health Organization. Obesity [Internet], 2014. Available: https://www.afro.who.int/health-topics/obesity

8 Klingberg S, Draper CE, Micklesfield LK, et al. Childhood obesity prevention in Africa: a systematic review of intervention effectiveness and implementation. Int J Environ Res Public Health 2019;16:1212.

9 Chin S-H, Kahathuduwa CN, Binks M. Physical activity and obesity: what we know and what we need to know. Obes Rev 2016;17:1226-44.

10 Chooi YC, Ding C, Magkos F. The epidemiology of obesity. Metabolism 2019;92:6-10.

11 Vecchié A, Dallegri F, Carbone F, et al. Obesity phenotypes and their paradoxical association with cardiovascular diseases. Eur J Intern Med 2017;2018:6-17.

12 Seravalle G, Grassi G. Obesity and hypertension. Pharmacol Res 2017;122:1-7.

13 Jacoby E, Grajeda R, Contreras A, et al. The epidemic of childhood obesity in the Americas must be stopped: governmental and PAHO leadership are crucial. Int J Obes Suppl 2013;3:S15-17.

14 Mittendorfer B. Origins of metabolic complications in obesity: adipose tissue and free fatty acid trafficking. Curr Opin Clin Nutr Metab Care 2011;14:535-41.

15 Harris J, Chisanga B, Drimie S, Harris J, et al. Nutrition transition in Zambia: changing food supply, food prices, household consumption, diet and nutrition outcomes. Food Secur 2019;11:371-87.

16 Fox A, Feng W, Asal V. What is driving global obesity trends? Globalization or "modernization"? Global Health 2019;15:1-16.

17 Hills AP, Byrne NM, Lindstrom R, et al. 'Small changes' to diet and physical activity behaviors for weight management. Obes Facts 2013;6:228-38.

18 Rush E, Yan M. Evolution not revolution: nutrition and obesity. Nutrients 2017:9:519-8.

19 Lien AS-Y, Tsai J-L, Lee J-T, et al. A systematic review and metaanalysis of the effect of lifestyle modification on metabolic control in overweight children. Evid Based Complement Alternat Med 2017;2017:1-12.

20 Scott A, Ejikeme CS, Clottey EN, et al. Obesity in sub-Saharan Africa: development of an ecological theoretical framework. Health Promot Int 2013;28:4-16.

21 Romieu I, Dossus L, Barquera S, et al. Energy balance and obesity: what are the main drivers? Cancer Causes Control 2017;28:247-58.

22 Nantel J, Mathieu M-E, Prince F. Physical activity and obesity: biomechanical and physiological key concepts. J Obes 2011;2011:1-10.

23 Liu Z, Xu H-M, Wen L-M, et al. A systematic review and metaanalysis of the overall effects of school-based obesity prevention interventions and effect differences by intervention components. Int J Behav Nutr Phys Act 2019;16:1-12.

24 Wiklund P. The role of physical activity and exercise in obesity and weight management: time for critical appraisal. J Sport Health Sci 2016;5:151-4.

25 van der Ploeg HP, Hillsdon M. Is sedentary behaviour just physical inactivity by another name? Int J Behav Nutr Phys Act 2017;14:1-8.

26 Wright SM, Aronne LJ. Causes of obesity. Abdom Radiol 2012;37:730-2.

27 Arksey H, O'Malley L. Scoping studies: towards a methodological framework. Int J Soc Res Methodol 2005;8:19-32.

28 Tricco AC, Lillie E, Zarin W, et al. R ESEARCH and R EPORTING $M$ ETHODS PRISMA extension for scoping reviews (PRISMA-ScR): checklist and explanation, 2018.

29 World Health Organization. Growth reference 5-19 years. 19, 2020. 